\title{
Integrating and Inspecting Combined Behavioral Profiling and Social Network Models in Destiny
}

\author{
André Rattinger ${ }^{1}$, Günter Wallner ${ }^{2}$, Anders Drachen ${ }^{3}$, Johanna Pirker ${ }^{1}$, and \\ Rafet $\mathrm{Sifa}^{4}$ \\ ${ }^{1}$ Graz University of Technology \\ andre.rattinger@gmail.com, jpirker@iicm.edu \\ 2 University of Applied Arts Vienna \\ guenter . wallner@uni-ak.ac.at \\ 3 Aalborg University \& The Pagonis Network \\ drachen@hum.aau.dk \\ 4 Fraunhofer IAIS \\ rafet.sifa@iais.fraunhofer.de
}

\begin{abstract}
In this paper two key venues of investigation in game analytics are combined: behavioral profiling and social network analysis. Both venues of research are well developed but combined they permit pattern evaluation across player performance and networks. Here, competitive networks covering almost 3.5 million players of the hybrid online shooter game Destiny are developed and combined with behavioral profiles based on match performance metrics and defined using archetypal analysis. The profiles are embedded in the networks along with other performance indicators for Destiny players. The social behavior of different archetypes is described. Network visualizations are presented which target the problem of making dense networked results actionable.
\end{abstract}

Keywords: Social Network Analysis, Destiny, Network Visualization, Multi-Player, Profiling, Game Analytics

\section{Introduction}

With the introduction of telemetry tracking in digital games, game analytics has become a cornerstone of game development [18]. Visualization of behavioral analysis on high-dimensional datasets is challenging because of the typical complex behavioral phenomena in games $[2,6,8,11,18,26]$. This challenge is a well-described problem in game analytics, where major commercial titles can give rise to thousands of different features [11]. To counter this problem a variety of methods have been adopted and adapted from other domains. Of these, behavioral profiling plays an established role. The focus here has been on condensing varied, volatile and high-volume data into condensed profiles which encapsulate player behavior and highlight key patterns of use for the specific purpose. The location of patterns in the behavior of players, and how to translate these to business action, using them to inform game AI or informing human behavior 
research, remains however a major line of inquiry [11]. The specific purpose of player profiling varies substantially, from top-down explorative analysis to hypothesis-testing $[2,11]$. Multiple examples have been described across a variety of games and using a variety of techniques, with unsupervised techniques such as clustering being the most common $[2,11,17]$. Cluster models allow segments to be developed which can describe the behavior of players according to specific behaviors and are driven by specific research questions, and they can be translated into behavioral descriptions of the different player segments. Behavioral profiling is notably useful in persistent online games, where the success of a title relies on its ability to keep a population of users engaged longitudinally and thus requires constant monitoring. This is also the situation in Destiny, a hybrid online shooter game which forms the case for the work presented here. Destiny is a persistent online game and the to date most expensive digital title to be developed worldwide, with a player base of roughly 30 million active players. As a hybrid title blending design aspects of multiple genres and featuring varied gameplay, e.g., multiple modes of play, Destiny forms an ideal basis for evaluating new frameworks and models for investigating player behavior.

Behavioral profiling work in games tends to focus on individual players, ignoring the connections between players, which are instead treated separately through social network analysis (SNA) [12]. From the perspective of SNA in games, the information about the players is generally limited, and the focus is instead on the links that connect players. This means that SNA typically views users as users, and ties minimal contextual information to the nodes of a network (demographics forming a common exception). In SNA, pattern recognition is as important as in behavioral profiling, and cluster analysis is used for defining groups and patterns in networks $[5,12,13]$.

For behavioral profiling and SNA, a central challenge rests in the visualization of the results of analysis. In both instances, data and the patterns in them need to be presented to a user such that they are interpretable and actionable $[11,12]$. In this paper, a step is taken towards combining game-based behavioral profiling and game-based SNA. This, however, means that the visualization problem is amplified as the relative scope of analysis is increased. However, combining these two lines of investigation in games research has the advantage of providing a framework for exploring in-game behavior of players along with the connections between players. This permits a more detailed understanding of the individual player, in essence providing a lens that permits the observation of network behavior as well as general in-game behavior. This, in turn, informs the evaluation of design, engagement analysis, monetization decisions and similar factors in games.

Contribution In this paper, game-based SNA, behavioral profiling, and data visualization is combined for the purpose of investigating the network behavior of competitive Destiny players as a function of the patterns in their performance, and ultimately develop novel visualizations that aim at making the results of combined SNA-profiling analysis interpretable by game designers and the 
Destiny players themselves. Towards this, competitive networks are constructed based on data from the Player-versus-Player $(\mathrm{PvP})$ game modes, covering almost 3.5 million players. Using archetypal analysis, behavioral profiles are defined for each player, based on a range of performance indicators which includes information about behavior with the different weapon classes in Destiny. Given the shooter-type nature of the game, weapon performance is a key indicator of player skill. The profiles are embedded in the competitive networks and used to analyze the player network as a function of profiles. Furthermore, other metrics are integrated in separate analyses and visualized.

\section{Related Work}

The work presented here builds on previous work in three domains: 1) behavioral profiling in games; 2) social network analysis in games; 3) visualization of complex behavioral datasets and networks. For reasons of space this section will focus on key related work.

Behavioral Profiling in Games: Cluster analysis is a method for dimensionality reduction and pattern recognition and has been readily applied across disciplines. As an unsupervised method, it permits the exploration of data and can identify groups of players with similar behaviors or detect features that constitute such behaviors $[2,23]$. Behavioral profiling in game analytics has explored a variety of cluster models, including a few comparative studies $[2,10]$. There is no standard for which model to employ in which situation, but previous work has highlighted that various models are useful for different situations and problems $[2,10]$. For example, Thurau and Bauckhage [24] explored the evolution of guilds in World of Warcraft using matrix factorization which provided condensed views on how guilds change their composition over time. For example, Sifa et al. [22] identified clusters of players based on their relative playtime distribution across games on the Steam distribution platform using k-means clustering. Thawonmas and Iizuka [23] employed multidimensional scaling (CMDS) and KeyGraph to generate visualizations of player clusters. Drachen et al. [8] employed simplex volume maximization and $\mathrm{k}$-means, developing behavioral profiles for two games. Normoyle and Jensen [17] used Bayesian Clustering. Moving into spatio-temporal clustering, Bauckhage et al. [3] developed waypoint graphs and adopted DEDICOM for behavior-based partitioning of player trajectories. Archetypal analysis (AA) was adopted by Drachen et al. [10] and Sifa et al. [20], who noted the desirable properties of the model for investigating extremal behaviors and permitting soft clustering, i.e. the expression of behavioral profiles in terms of their belongingness to multiple cluster centers. In this paper, AA is adopted as the cluster model.

Social Network Analysis: SNA of relations between people has in recent years become a commonly employed tool, with large-scale online platforms such as Facebook and Twitter providing a direct vehicle for investigation. Prior work has targeted not only analysis of social networks themselves, but also their potential for recommendation and prediction of user behavior. SNA has clear application 
in games, where the value of the different types of relations between players that exist in multi-player or massively multi-player online games to, e.g., player retention and user experience has been shown in a number of studies (e.g., [27]).

Due to the hybrid nature of Destiny there is no prior work that is directly comparable with the study presented here. Furthermore, the integration of detailed behavioral features into the network structure is also unexplored. Furthermore, most previous work has focused on analyzing social structures in persistent online communities, rather than competitive team-based games. However, Iosup et al. [16] examined networks in DOTA and StarCraft with a specific focus on modeling social structure and network robustness toward retention. Similarly, Jia et al. [13] introduced networks generated from team-based match data, including networks based on players being in the same match, on the same or different side in a match.

Visualization of Player Behavior: Visualization of player behavior in game analytics has covered a variety of goals but the majority of the work is focused on the spatio-temporal components of behavior. Wallner and Kriglstein [26] provide a recent review of the area, noting the wide range of techniques employed. Drachen and Schubert [9] reviewed spatio-temporal visualizations in use in game development. In general, the emphasis in the context of games is on pattern recognition and visualization of these patterns in a way that is actionable to the relevant stakeholder.

In network analysis, a wide range of visualizations have been proposed, but with an overall focus on link properties as compared to node properties. There are two common forms of display (cf. [12]): 1) The most common visualization is based on node-link diagrams where the nodes represent the social actors, and lines connections between them; 2) Matrices, where rows and columns represent social actors and various coal connections linking them. Recent years have seen the convergence of SNA and visualization, combined with interaction, but remains generally focused on network properties, including labeling, rather than node properties [7].

\section{Background: Destiny}

Destiny is a science-fiction themed game where players need to defend the Earth from various alien threats, taking on the role of Guardians. Humanity is reduced to one last city, and it is up to the players to make sure the city stays safe, while working for the overall goal of reviving a Deathstar-sized sphere being called the Traveler, who protected human civilization in the past but currently lays dormant. Players journey to different planets, complete missions, daily events, and perform a variety of different tasks to build up their characters and help eliminate the alien threat. Destiny is a hybrid digital game that blends features from a number of traditional game genres but which is first and foremost a shooter. The main components of the gameplay is focused on tactical singleplayer or small-team combat, and the number of weapons, modifications and customizations in the game is staggering. 
Weapons are divided into over a dozen different classes, each specialized for specific situations. For example, sniper rifles offer high power at long range, but are virtually useless at close range and require player skill to aim precisely. The utility of different weapon classes varies between game modes and in-game situations. Notably, the amount of damage that players in $\mathrm{PvP}$ can absorb can be dramatically different from the damage it takes to eliminate tough aliens in Player-versus-Environment $(\mathrm{PvE})$ mode. In general, players are free to switch between any combination of weapon types, allowing for adjustment to in-game scenarios while at the same time reflecting individual behavior and preferences. Weapon-related performance metrics thus form one possible starting point for behavioral profiling of Destiny players, which targets the core shooter gameplay. Here, weapon class data is augmented with further performance metrics to provide a broad profile for developing profiles focusing on player performance in the game.

For reasons of limited space, the focus here is on those parts of Destiny that relate directly to $\mathrm{PvP}$ combat, but it should be pointed out that the game also has multiple PvE modes. PvP in Destiny is played in the Crucible - a central hub for the various $\mathrm{PvP}$ match types. These are commonly played in so-called fireteams comprised of 3 players on each side. Typically players can bring three different weapons to a $\mathrm{PvP}$ match. In the Crucible, players can earn medals (awards), points, and in-game currency by accomplishing tasks, winning matches, performing specific tasks, or feats of skill. Destiny features three different player classes, each with distinct abilities. Each class is divided into three subclasses. Players can level up their characters until level 40, unlocking new abilities and gradually becoming more powerful along with obtaining new weapons and other equipment through gameplay. In both $\mathrm{PvE}$ and $\mathrm{PvP}$ game modes, players are rewarded with new weapons and items through random drops or by completing specific tasks.

\section{Behavioral Profiling with Extremes}

Considering the large scale nature of today's behavioral datasets and the vast diversity of play-styles, finding profiles gives us the flexibility to manage, understand, and group the common behavioral patterns for the process of informed decision making. In this section we give a brief overview of Archetypal Analysis and it's use for player profiling. Archetypal analysis [1] is a constrained matrix factorization method that allows for soft clustering and is based on representing the data points as a convex combination of extreme datapoints that are called archetypes. Formally, given a data matrix $\mathbf{X} \in \mathbb{R}^{m \times n}$ we aim to find factors minimizing the matrix norms

$$
\|\mathbf{X}-\mathbf{X B A}\|^{2}=\|\mathbf{X}-\mathbf{Z A}\|^{2}
$$

where $\mathbf{Z} \in \mathbb{R}^{m \times k}$ represents the archetypes, $\mathbf{A} \in \mathbb{R}^{k \times n}$ and $\mathbf{B} \in \mathbb{R}^{n \times k}$ are column stochastic and represent the mixing coefficients respectively for archetypes 
and datapoints that represent the archetypes. Various techniques have been proposed to find appropriate factors for the above decomposition and its approximations. Examples of these include methods using pure alternating least square updates [1], active set updates [4], or distance geometry [25]. In the context of game analytics, archetypal analysis is used to profile players $[8,20,15]$, build recommender systems [21], and generate human like bots [19]. In this study, as proposed in $[8,20]$, we will concentrate on the use of archetypal analysis for finding player profiles in Destiny, in which we are interested in finding prototypical players that are encoded in $\mathbf{Z}$ to define particular player styles and belongingness coefficients represented in $\mathbf{A}$ showing how much each player belongs to the profiles defined in $\mathbf{Z}$.

\section{$5 \quad$ Player Networks and Characteristics}

In multi-player games players interact, play together or against each other, build different in-game groups and are thus forming complex relationships and ingame structures. We can map such relationships to networks to measure and analyze these interactions. To illustrate player interactions in Destiny we can build networks based on different forms of interactions. One way to illustrate in-game relationships is to build an undirected, weighted graph based on the information how often players play matches against or - as done in this paper with the same players. In the network, players are represented as nodes, and their match relationships are modeled as weighted (based on the number of matches played together) links.

\subsection{Network Measures}

For the following analysis three different networks were created. Links for all networks are built between players that played matches together in the same team. The first network (main network, MN) describes the social structure of all $3,362,636$ players based on match information of 930,720 matches. The second network (connected network, CN) illustrates a fully-connected friendship network based on three well-connected players. These players were used as starting point for a breadth-first-search and connected players were added to this network up to a network size of 1,000 nodes. 11 players were removed from the resulting network because of missing weapon information. The third network (random network, RN) was built based on 10,000 randomly picked players. Table 1 gives an overview of the network characteristics of the three player networks. The largest connected component $(L C C)$ describes the largest self-contained subgraph in the network. A large LCC refers to a well-connected main graph. Looking at the number of nodes and links in the LCC the MN is well-connected, $\mathrm{CN}$ is fully connected (as designed), and RN is barely connected. The degree (k) of a player refers to the number of links to other players. The average degree refers to the average number of links to other players. The diameter describes the longest of the shortest paths between two nodes and can be used to illustrate the linear size of a network. 
Table 1. Methodological comparison of the three networks, (a) the main network with over 3 million players, (b) a well-connected network, and (c) a random selection of 10,000 players ( $\mathrm{LCC}=$ largest connected component).

\begin{tabular}{rccc}
\hline & MN & CN & RN \\
\hline Nodes & $3,362,636$ & 989 & 7,479 \\
Nodes in LCC & $3,347,226$ & 989 & 407 \\
Links & $22,638,062$ & 2,321 & 1,601 \\
Links in LCC & $22,614,017$ & 2,321 & 432 \\
Network size & $3,362,596$ & 989 & 1,991 \\
Average degree & 13 & 4 & 0.428 \\
Diameter & 15.0 & 4.0 & 23 \\
\hline & MN = main network, CN = connected network, RN = random network
\end{tabular}

Table 2. Overview of the different archetypes

\begin{tabular}{ll}
\hline Archetype & Description \\
\hline Ranged Elites (AT1) & $\begin{array}{l}\text { pretty good scores, auto-rifle focus, higher killing spree, } \\
\text { unique precision kills, kill/death ratio, and win rate } \\
\text { than AT3 } \\
\text { melee focused, medium performance, win rate similar } \\
\text { to AT4 } \\
\text { Melee (AT2) }\end{array}$ \\
Mighed Weapon Elites (AT3) & $\begin{array}{l}\text { weapon scores except for auto-rifle, slightly lower win } \\
\text { rate } \\
\text { medium performance, heavy use of shotgun, some } \\
\text { melee } \\
\text { low performance everywhere }\end{array}$ \\
Newbies (AT5) &
\end{tabular}

\subsection{Archetypes}

Using archetypal analysis (AA) we identified five player archetypes (see Table 2) based on their most distinguished properties. 15 features from the Destiny dataset were selected (such as precision kills, scores, medals, kill/death ratio, and weapon preference) to be used with AA. Those features describe the in-game behavior and success of Destiny players in $\mathrm{PvP}$ matches. Figure 1 illustrates the five archetype profiles across the 15 selected behavioral features. Those features can be split into success-based features and weapon usage features. It further illustrates the varying weapon usage behavior between the archetypes. Table 3 lists the differences in weapon usage between the archetypes for the different weapon ranges (short-, mid-, and long-range). The majority of weapon use for each archetype focuses on short and mid-range weapons, but there are a few exceptions. For instance, some players use long-range weapons almost exclusively. 


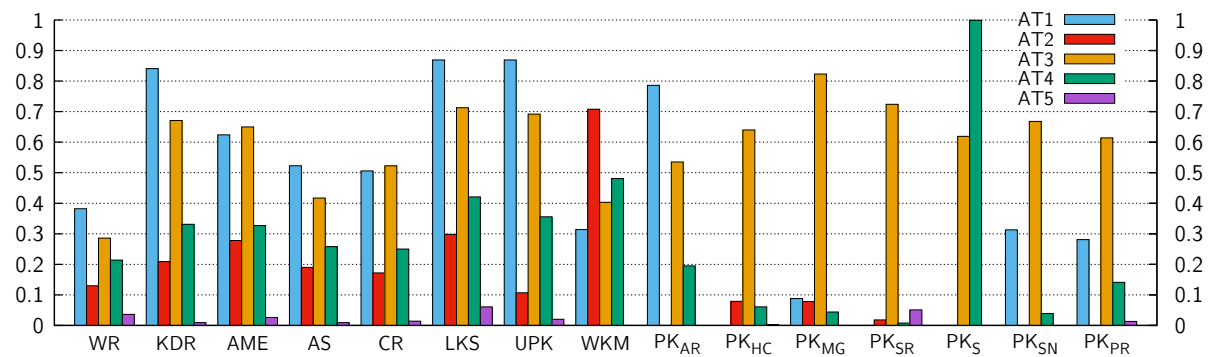

Fig. 1. Archetype profiles for Destiny across behavioral features. WR = win rate, $\mathrm{KDR}=$ kill $/$ death ratio, $\mathrm{AME}=$ all medals earned, $\mathrm{AS}=$ average score, $\mathrm{CR}=$ combat rating, $\mathrm{LKS}=$ longest killing spree, $\mathrm{UPK}=$ unique precision kills, $\mathrm{WKM}=$ weapon kills melee, $\mathrm{PK}=$ precision kills with auto rifle $(\mathrm{AR})$, hand cannon $(\mathrm{HC})$, machine gun $(\mathrm{MG})$, scout rifle $(\mathrm{SR})$, shotgun $(\mathrm{S})$, sniper rifle $(\mathrm{SN})$, and pulse rifle $(\mathrm{PR})$

Table 3. Weapon use of the five different archetypes

\begin{tabular}{cccc}
\hline Archetype & Short-range & Mid-range & Long-range \\
\hline AT1 & $\mathrm{M}=0.45, \mathrm{SD}=0.38$ & $\mathrm{M}=0.45, \mathrm{SD}=0.32$ & $\mathrm{M}=0.094, \mathrm{SD}=0.49$ \\
AT2 & $\mathrm{M}=0.41, \mathrm{SD}=0.28$ & $\mathrm{M}=0.46, \mathrm{SD}=0.23$ & $\mathrm{M}=0.13, \mathrm{SD}=0.38$ \\
AT3 & $\mathrm{M}=0.42, \mathrm{SD}=0.12$ & $\mathrm{M}=0.45, \mathrm{SD}=0.10$ & $\mathrm{M}=0.13, \mathrm{SD}=0.15$ \\
AT4 & $\mathrm{M}=0.45, \mathrm{SD}=0.17$ & $\mathrm{M}=0.46, \mathrm{SD}=0.15$ & $\mathrm{M}=0.095, \mathrm{SD}=0.20$ \\
AT5 & $\mathrm{M}=0.48, \mathrm{SD}=0.11$ & $\mathrm{M}=0.42, \mathrm{SD}=0.10$ & $\mathrm{M}=0.097, \mathrm{SD}=0.14$ \\
\hline
\end{tabular}

In order to investigate if players belonging to a specific archetype have a tendency to play with the same group of players or if they rather play with random players we tried to map the archetypes to social network metrics. For that purpose, we calculated the weighted arithmetic mean $\bar{a}$ to determine how likely it is that a player plays with the same group of players, that is sum of weights $/ k$. However, we could not find notable differences between the behavior of players from certain archetypes when matching up with team members, with $\bar{a}$ being 1.226 (AT1), 1.225 (AT2), 1.219 (AT3), 1.224 (AT4), and 1.233 (AT5).

\section{Visualizations and Network Integration}

Figure 2 shows a node-link visualization of the $\mathrm{CN}$ network which represents each node as a donut chart. This donut chart denotes the weapon usage (short-, mid-, and long-range) of a player. The size of a node is proportional to the number of matches played, that is, players playing more often are represented by larger nodes. Edges show which players played in the same team. The thickness of the edges corresponds to the number of matches played together, i.e. players who play together more often are connected by thicker edges. Edges are rendered semitransparent whereas the degree of transparency is weighted based on the number of matches in order to accentuate stronger relationships. As can be 


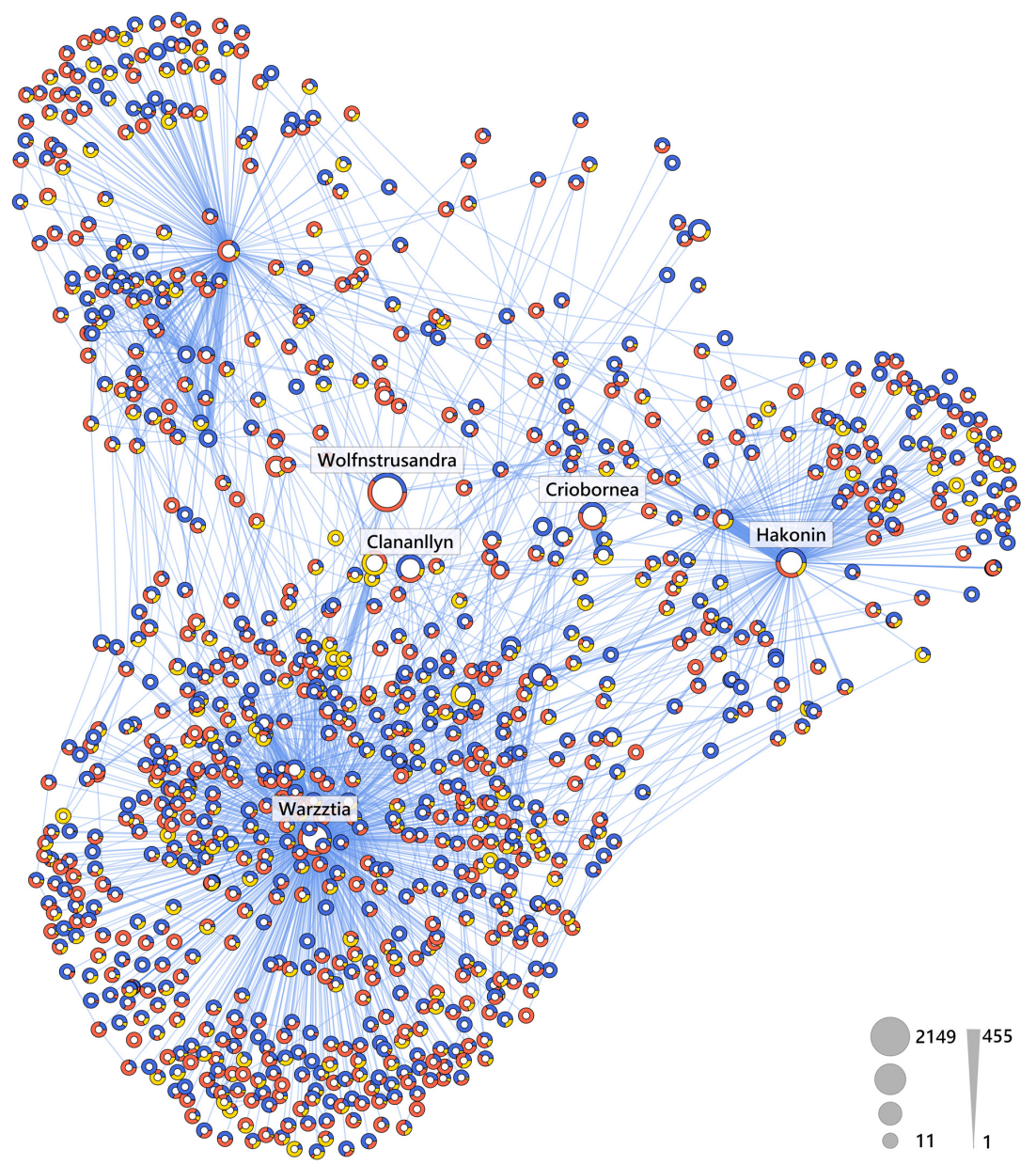

Fig. 2. Visualization of the fully-connected friendship network with 964 nodes. Donut charts at each node show the weapon usage for the individual players ( short range, mid range, long range). Labels are shown for players who played more than 1000 matches. Player names were anonymized.

inferred from Figure 2 players in this particular network prefer short-range ( blue) and mid-range weapons ( $\square$ red) over long distance weapons ( $\square$ yellow).

Figure 3 depicts the result of the AA for the random network (based on a subset of 7479 players). The degree of membership to each of the five archetypes is depicted through pie-charts. The spatial arrangement of nodes has been derived using multidimensional scaling (e.g., [14]) such that players with similar archetype membership are placed in proximity of each other. To this end, the probabilities of belonging to each archetype were treated as a five-dimensional feature vector. The similarity of two players with feature vectors $\mathbf{A}$ and $\mathbf{B}$ 


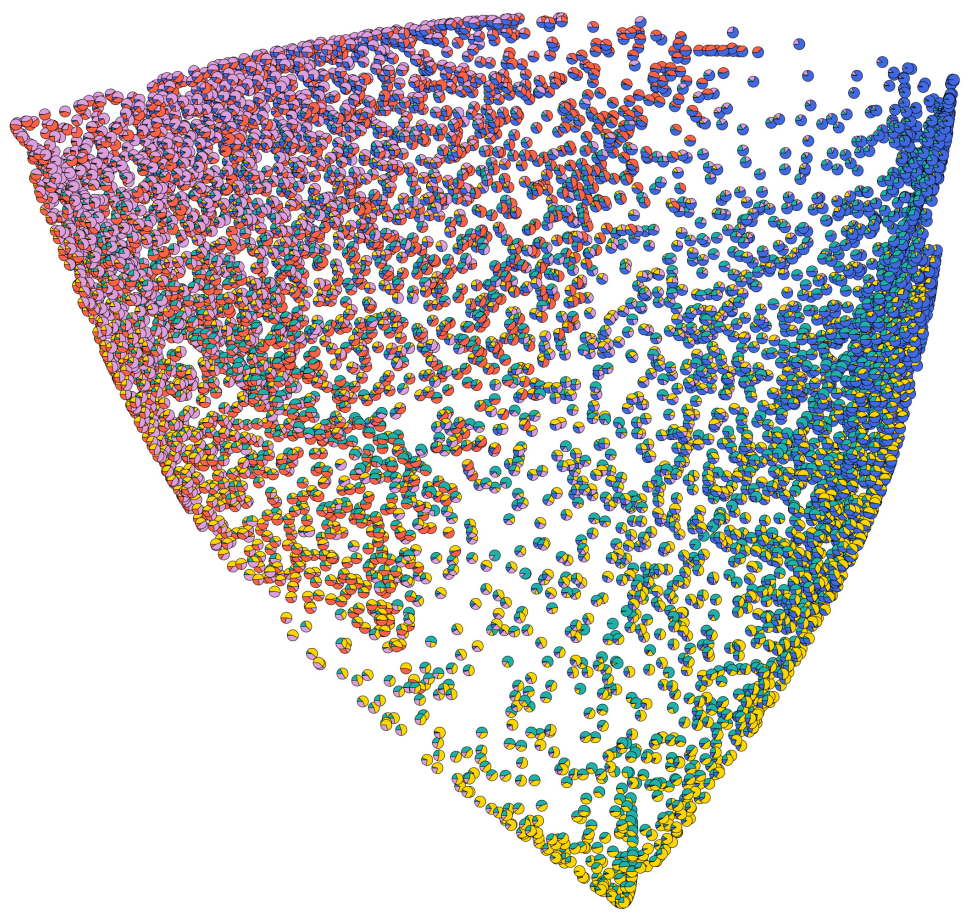

Fig. 3. Players' degree of membership to each of the five archetypes ( AT1 - Ranged Elites, AT2 - Melee, $\square$ AT3 - Mixed Weapon Elites, AT4 - Short Range, AT5 - Newbies).

was then measured using cosine similarity (see, e.g., [21]), that is, $s_{A B}=\mathbf{A}$. $\mathbf{B} /\|\mathbf{A}\|\|\mathbf{B}\|$. From Figure 3 it can be observed that players form three major groups: (i) players mainly belonging to the novice archetype (upper right corner), (ii) players mainly being considered melee focused but also sharing traits of the ranged elites and to a lesser degree of novices (lower right corner), and (iii) players primarily sharing characteristics of the mixed weapon elites and short range archetypes (upper left corner) with some of them exhibiting characteristics of either the novices, ranged elites, or melee archetype. The latter group also accounts for the largest of the three groups in the investigated network. Players sharing traits of four or even five archetypes are more uncommon as reflected by the sparse area in the center of the figure. While this indicates specialization to a certain extent it is also apparent that players can rarely be attributed to a single archetype.

\section{Conclusion and Discussion}

In this paper behavioral profiles have been constructed based on performance telemetry data from the PvP activities of 10,000 players, randomly selected from 
those who had played for at least two hours, of the persistent online FPS game Destiny, using Archetypal Analysis. A five-cluster solution was found, which showcases how performance-based profiling in the PvP component of Destiny can be closely related to the choices players make with respect to which weapons they use, as well as their relative skill, as expressed, e.g., via their kill/death ratios. Each of the players can be expressed in terms of their degree of membership to each archetype. The profiles have been embedded in a competitive player network covering the almost 3.5 million players that the 10,000 are connected to. The competitive network maps the tendency of people in Destiny to play with the same people or random groups across the various $\mathrm{PvP}$ modes of the game. In addition, a number of behavioral metrics has been examined in terms of their distribution in the network, notably weapon use distribution, which is a key indicator in Destiny as the main gameplay revolves around combat.

Future work will focus on evaluating player performance and network structure as a function of the number of players $\mathrm{PvP}$ matches. Initial dataset analysis indicated a relationship between performance metrics and the number of matches played. Furthermore, players tend to gravitate from using specific weapon classes during early matches to other weapon classes after having played many PvP matches. Both of these patterns will be verified and investigated in future work. Furthermore, the current work developed profiles for 10,000 players in a 3.5 million player network, and it is therefore an obvious next step to scale up the profiling to all the players in the network. This would substantially increase the sample size but also requires algorithms that scale well to such large datasets. Finally, the visualizations presented here will be further evaluated and expanded on, and a web-based interface developed which will target both game designers and players of competitive multi-player matches for performance evaluation.

Acknowledgments: The authors would like to extend their sincere gratitude to Bungie for making detailed behavioral telemetry from Destiny available.

\section{References}

1. Adele Cutler, L.B.: Archetypal analysis. Technometrics 36(4), 338-347 (1994)

2. Bauckhage, C., Drachen, A., Sifa, R.: Clustering game behavior data. IEEE Transactions on Computational Intelligence and AI in Games 7(3), 266-278 (2015)

3. Bauckhage, C., Sifa, R., Drachen, A., Thurau, C., Hadiji, F.: Beyond heatmaps: Spatio-temporal clustering using behavior-based partitioning of game levels. In: IEEE Conference on Computational Intelligence and Games. pp. 1-8 (2014)

4. Bauckhage, C., Thurau, C.: Making archetypal analysis practical. In: Denzler, J., Notni, G., Süße, H. (eds.) Proceedings Pattern Recognition: 31st DAGM Symposium. pp. 272-281. Springer Berlin Heidelberg (2009)

5. van de Bovenkamp, R., Shen, S., Iosup, A., Kuipers, F.: Understanding and recommending play relationships in online social gaming. In: Fifth International Conference on Communication Systems and Networks (COMSNETS). pp. 1-10 (2013)

6. Cleveland, W.S.: Visualizing Data. Hobart Press (1993) 
7. Correa, C.D., Ma, K.L.: Visualizing social networks. In: Aggarwal, C.C. (ed.) Social Network Data Analytics, pp. 307-326. Springer US (2011)

8. Drachen, A., Sifa, R., Bauckhage, C., Thurau, C.: Guns, swords and data: Clustering of player behavior in computer games in the wild. In: IEEE Conference on Computational Intelligence and Games. pp. 163-170 (2012)

9. Drachen, A., Schubert, M.: Spatial game analytics. In: Seif El-Nasr, M., Drachen, A., Canossa, A. (eds.) Game Analytics: Maximizing the Value of Player Data, pp. 365-402. Springer London (2013)

10. Drachen, A., Thurau, C., Sifa, R., Bauckhage, C.: A comparison of methods for player clustering via behavioral telemetry. In: Proc. FDG. pp. 245-252 (2013)

11. Drachen, A., Thurau, C., Togelius, J., Yannakakis, G.N., Bauckhage, C.: Game data mining. In: Seif El-Nasr, M., Drachen, A., Canossa, A. (eds.) Game Analytics: Maximizing the Value of Player Data, pp. 205-253. Springer London (2013)

12. Freeman, L.C.: Visualizing social networks. Journal of Social Structure 1 (2000)

13. Jia, A.L., Shen, S., Bovenkamp, R.V.D., Iosup, A., Kuipers, F., Epema, D.H.J.: Socializing by gaming: Revealing social relationships in multiplayer online games. ACM Trans. Knowl. Discov. Data 10(2), 11:1-11:29 (2015)

14. Kruskal, J.B., Wish, M.: Multidimensional Scaling. Sage Publications (1978)

15. Lim, C.U., Harrell, D.F.: Revealing social identity phenomena in videogames with archetypal analysis. In: Proc. AISB Symp. on AI and Games (2015)

16. losup, A., van de Bovenkamp, R., Shen, S., Jia, A.L., Kuipers, F.: Implicit social networks in multiplayer online games. IEEE Internet Comp. 18(3), 36-44 (2014)

17. Normoyle, A., Jensen, S.T.: Bayesian clustering of player styles for multiplayer games. In: Proc. AAAI. pp. 163-169 (2015)

18. Seif El-Nasr, M., Drachen, A., Canossa, A. (eds.): Game Analytics - Maximizing the Value of Player Data. Springer London (2013)

19. Sifa, R., Bauckhage, C.: Archetypical motion: Supervised game behavior learning with archetypal analysis. In: IEEE Conference on Computational Intelligence in Games. pp. 1-8 (2013)

20. Sifa, R., Drachen, A., Bauckhage, C., Thurau, C., Canossa, A.: Behavior evolution in tomb raider underworld. In: IEEE Conference on Computational Intelligence in Games. pp. 1-8 (2013)

21. Sifa, R., Bauckhage, C., Drachen, A.: Archetypal game recommender systems. In: Proceedings of the 16th LWA Workshops: KDML, IR and FGWM. pp. 45-56 (2014)

22. Sifa, R., Drachen, A., Bauckhage, C.: Large-scale cross-game player behavior analysis on steam. In: Proc. AIIDE. pp. 198-204 (2015)

23. Thawonmas, R., Iizuka, K.: Visualization of online-game players based on their action behaviors. Int. J. Comput. Games Technol. 2008, 5:1-5:9 (2008)

24. Thurau, C., Bauckhage, C.: Analyzing the evolution of social groups in World of Warcraft. In: IEEE Conference on Computational Intelligence and Games. pp. 170-177 (2010)

25. Thurau, C., Kersting, K., Bauckhage, C.: Yes we can: Simplex volume maximization for descriptive web-scale matrix factorization. In: Proc. of the 19th ACM International Conference on Information and Knowledge Management. pp. 17851788. CIKM '10, ACM (2010)

26. Wallner, G., Kriglstein, S.: An introduction to gameplay data visualization. In: Lankoski, P., Björk, S. (eds.) Game Research Methods, pp. 231-250. ETC (2015)

27. Yee, N.: The demographics, motivations, and derived experiences of users of massively multi-user online graphical environments. Presence: Teleoper. Virtual Environ. 15(3), 309-329 (2006) 